\title{
Analysis of Optical Properties for Square, Circular and Hexagonal Photonic Crystal Fiber
}

\author{
Md. Bellal Hossain, Abdullah Al-Mamun Bulbul, Md. Abdul Mukit, Etu Podder \\ Electronics and Communication Engineering (ECE) Discipline, Khulna University, Khulna, Bangladesh \\ Email: md.bellal.ku@gmail.com
}

How to cite this paper: Hossain, M.B. Bulbul, A.A.-M., Mukit, M.A. and Podder, E. (2017) Analysis of Optical Properties for Square, Circular and Hexagonal Photonic Crystal Fiber. Optics and Photonics Journal, 7, 235-243.

https://doi.org/10.4236/opj.2017.711021

Received: October 24, 2017

Accepted: November 12, 2017

Published: November 15, 2017

Copyright () 2017 by authors and Scientific Research Publishing Inc. This work is licensed under the Creative Commons Attribution International License (CC BY 4.0).

http://creativecommons.org/licenses/by/4.0/

\begin{abstract}
This paper presents four rings square, circular, and hexagonal photonic crystal fiber (PCF) geometry for analyzing different optical properties in a wavelength ranging from $800 \mathrm{~nm}$ to $1600 \mathrm{~nm}$. These three types of geometry have been used for analyzing Effective area, Propagation constant, Confinement loss and Waveguide dispersion. Silica glass is chosen as background material and the cladding region is made of four air hole layers. COMSOL Multiphysics (v.5) software is used to simulate these proposed PCF geometries. From the numerical analysis, it is found that the effective area is small for hexagonal PCF geometry and large for square PCF geometry $\left(11.827 \mu \mathrm{m}^{2}, 10.588 \mu \mathrm{m}^{2}\right.$ and $9.405 \mu \mathrm{m}^{2}$ for square, circular, and hexagonal PCF geometry respectively). From the analysis, the Confinement loss is approximately zero at wavelength ranges from $800 \mathrm{~nm}$ to $1250 \mathrm{~nm}$ and approximately zero waveguide dispersion is achieved from $900 \mathrm{~nm}$ to $1500 \mathrm{~nm}$ for all the three PCF structures. Again, negative dispersion approximately $-30.354 \mathrm{ps} /(\mathrm{nm} \cdot \mathrm{km})$ is achieved for circular PCF structure at the wavelength of $900 \mathrm{~nm}$.
\end{abstract}

\section{Keywords}

Photonics Crystal Fiber, Effective Area, Waveguide Dispersion, Confinement Loss, COMSOL Multiphysics

\section{Introduction}

Photonic crystal fiber (PCF) is among the most special optical light guides. The core region always has high refractive index (silica glass) where the cladding region is usually provided by micro structured arrangement of air holes along the fiber [1]. Conventional fibers have harsh design rules as the core size is limited as well as limitation on material selection where light propagates through it using total internal reflection (TIR) concept [2], as a result light can propagate a small 
distance. But in photonic crystal fibers, the light propagation consequence is somewhat different [3]. To propagate maximum light through the core region requires high refractive index than cladding and this phenomenon achieves by adding micro structured array of holes in the clad region [4] [5]. According to the guiding mechanisms, PCFs are usually categorized into two types: index guiding and photonic band gap guiding. For both types of PCF, specific fiber properties like effective area [6], dispersion [7], nonlinearity [8] and birefringence [9], confinement loss [10], propagation constant [11] etc. can easily be varied by changing holes size, arrangement of spacing and shape. All these exclusive optical properties of PCFs have contributed to advance development of these structures in numerous uses in the fields of optical communications [7] [10], nonlinear optics [8] and high-power technology [12]. Effective refractive index is an important term for PCF which has relation with the evaluation of different properties of optical communication [13]. This refractive index is obtained in complex form which is varied with the wavelength of light in PCF. Again, this variation of refractive index also varies these important optical properties such as effective area, waveguide dispersion, confinement loss, propagation constant etc. [4]. Confinement loss depends on the imaginary value of effective refractive index and effective area, waveguide dispersion as well as propagation constant depend on the real part of effective refractive index. It is possible to design application oriented guiding properties, by tuning the diameter and pitch of the air holes in the cladding [14]. The large effective mode area PCFs are required in optical transmission systems [15] and they are required to support broadband optical transmission as well as to minimize the coupling losses through the fibers. A design of a highly nonlinear dispersion-shifted fiber (HNDSF) with an effective area of $9.3 \mu \mathrm{m}^{2}$ is offered [16], and this advanced HNDSF structure can offer also low attenuation, and bending losses. Besides, fiber dispersion and confinement loss also play very important roles in broadband communications systems. This is rigorously achieved by ensuring ultra-flattened dispersion characteristics of fibers [17] as well as low confinement loss [7]. Hence, there are only a number of published papers dealing with PCFs having both better effective area and dispersion-flattened characteristics at the same time [18] [19]. So, there is still scope to design PCF of different structures with low confinement loss as well as low dispersion.

The main goal of the work is to design four rings square, circular and hexagonal geometry as simple as possible for analyzing effective area, propagation constant, confinement loss, waveguide dispersion etc. in a wavelength ranges from $800 \mathrm{~nm}$ to $1600 \mathrm{~nm}$. These structures ensure small effective mode area, low dispersion and low confinement loss in a wide wavelength range and the proposed structures are relatively simpler than the existing designs.

\section{Materials and Methods}

In this thesis, we have designed photonic crystal fiber of square, circular and hex- 
agonal structure with four layers of air holes in COMSOL (version 5). The Silica glass $(n=1.46)$ material is used as a background material. We have taken four layers of air holes to guide the light in the core region.

The first (inner) layer is elliptical with diameter, $\mathrm{d} 1=0.35 \mathrm{um}$ in $\mathrm{x}$ axis and $0.7 \mathrm{um}$ in $\mathrm{y}$ axis. The other three layers are circular shape with diameter $\mathrm{d} 2=\mathrm{d} 3$ $=\mathrm{d} 4=1.4 \mathrm{um}$ and pitch, $\Lambda=2 \mathrm{um}$. A perfectly matched layer (PML) absorbing boundary condition is applied after the air hole layers.

Our proposed structures are simulated for same air hole diameter, pitch to evaluate the optical properties such as effective area, propagation constant, confinement loss, and waveguide dispersion under wavelength ranges from $800 \mathrm{~nm}$ to $1600 \mathrm{~nm}$. Effective area is the fiber area that covers transverse dimension of the fiber. A low effective area provides high density of power in the core region required for non-linear effects to be significant. The effective area can also be related to spot-size, with the Gaussian width w, though. The effective area can be calculated directly from COMSOL Multiphysics. The effective mode area $A_{\text {eff }}$ is established [6]:

$$
A_{\text {eff }}=\left(\iint\left|E^{2}\right| \mathrm{d} x \mathrm{~d} y\right)^{2} / \iint\left|E^{4}\right| \mathrm{d} x \mathrm{~d} y
$$

Here, the effective mode area $A_{\text {eff }}$ in $\mu \mathrm{m}^{2}$ and electric field amplitude is $E$ in the medium.

The propagation constant of an electromagnetic wave is a measure of the change undergone by the amplitude of the wave as it propagates in a given direction. The propagation constant $\beta$ is given by [11]:

$$
\beta=n_{\text {eff }} 2 \Pi / \lambda
$$

where, $n_{\text {eff }}$ is the effective refractive index and $\lambda$ is the wavelength of the input light.

Confinement loss is one of the most important parameters in the fiber transmission. The loss in light confinement due to the periodic arrangement of cladding in PCF is called the confinement loss. Confinement loss is calculated from the following equation [14]:

$$
L=8.686 K_{o} I_{m}\left(n_{\text {eff }}\right)
$$

where, $K_{0}=2 \pi / \lambda$ and it is the propagation constant in free space and $I_{m}\left(n_{e f f}\right)$ is the imaginary part of effective refractive index.

When the fraction of light power propagating through the cladding region faster than the core region, then waveguide dispersion occurs. Waveguide dispersion is calculated from the following equation [14]:

$$
D=-\lambda / c\left(\mathrm{~d}^{2} n_{\text {eff }} / \mathrm{d} \lambda^{2}\right)
$$

where, $\lambda$ is the wavelength and $c$ is the velocity of light in free space.

At the first step, we used COMSOL Multiphysics software for creating four rings square, circular, and hexagonal photonic crystal fiber structures. After defined all parameters, the simulation is run. After simulating, we got the structur- 
al view of the respective design, confinement of the electric field in the core region of those respective designs. COMSOL Multiphysics software simulation gives us the complex effective refractive index which has a real part and an imaginary part. In the second step, we used these values to calculate effective area, propagation constant, dispersion and confinement loss using Microsoft Office Excel, and MATLAB was used to plot the outcome.

\section{Results and Discussions}

COMSOL Multiphysics software simulation gives us the fundamental effective refractive index data for square, circular and hexagonal photonics crystal fiber structure at a wavelength ranges from $800 \mathrm{~nm}$ to $1600 \mathrm{~nm}$. For each structure, the silica glass $(n=1.46)$ material is used as a background material and we have taken four layers of air holes.

The first (inner) layer is elliptical shape with diameter, $\mathrm{d} 1=0.35 \mathrm{um}$ in $\mathrm{x}$ axis and $0.7 \mathrm{um}$ in y axis. The other three layers are circular shape with diameter, $\mathrm{d} 2$ $=\mathrm{d} 3=\mathrm{d} 4=1.4 \mathrm{um}$ and pitch, $\Lambda=2 \mathrm{um}$. We tried to focus on the variation of effective area, confinement loss, waveguide dispersion, and propagation constant etc. due to change of structure of PCFs.

Figures 1-3 represent transverse geometry and fundamental mode field for the square, circular and hexagonal PCF geometry.

Figure 4 illustrated the effective area variation with respect to wavelength from $800 \mathrm{~nm}$ to $1600 \mathrm{~nm}$ for square, circular and hexagonal geometry. The effective area increases with the increases of wavelength for all the three structures. The effective area is large for square PCF geometry and small for hexagonal PCF geometry. The effective area is found $11.827 \mu \mathrm{m}^{2}, 10.588 \mu \mathrm{m}^{2}, 9.405 \mu \mathrm{m}^{2}$ for square, circular, and hexagonal PCF geometry respectively at wavelength 1550 nm.

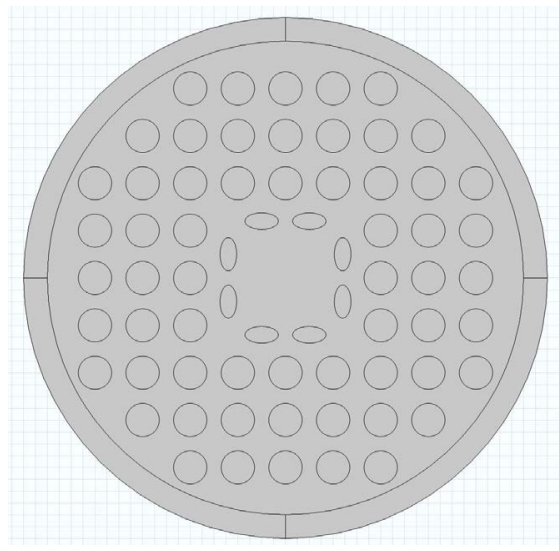

(a)

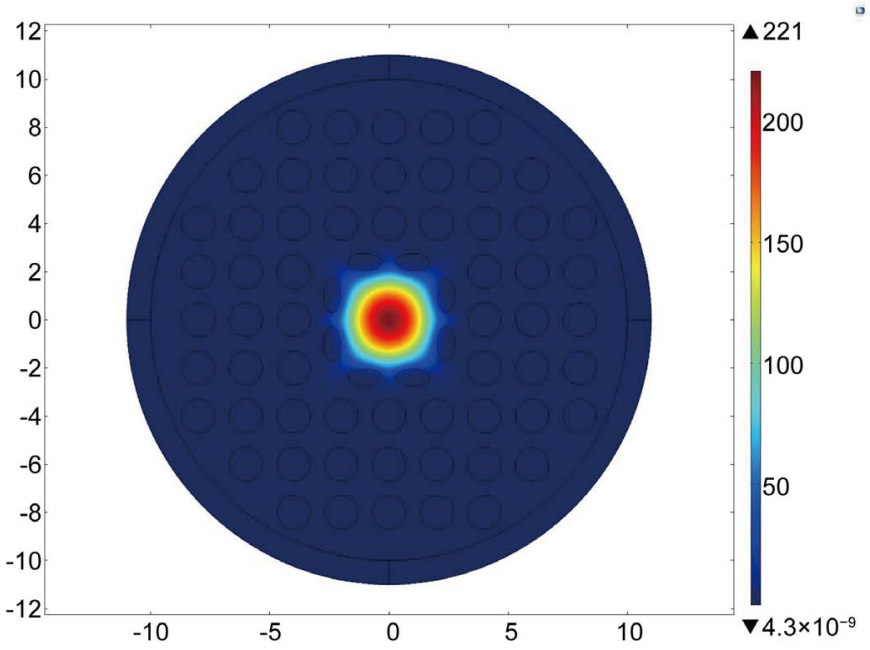

(b)

Figure 1. Analyzed square PCF model in COMSOL Multiphysics: (a) Transverse geometry; and (b) Fundamental mode field of the square PCF geometry. 


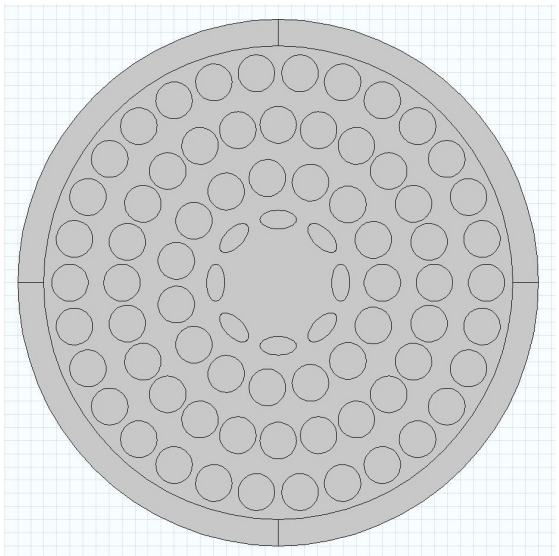

(a)

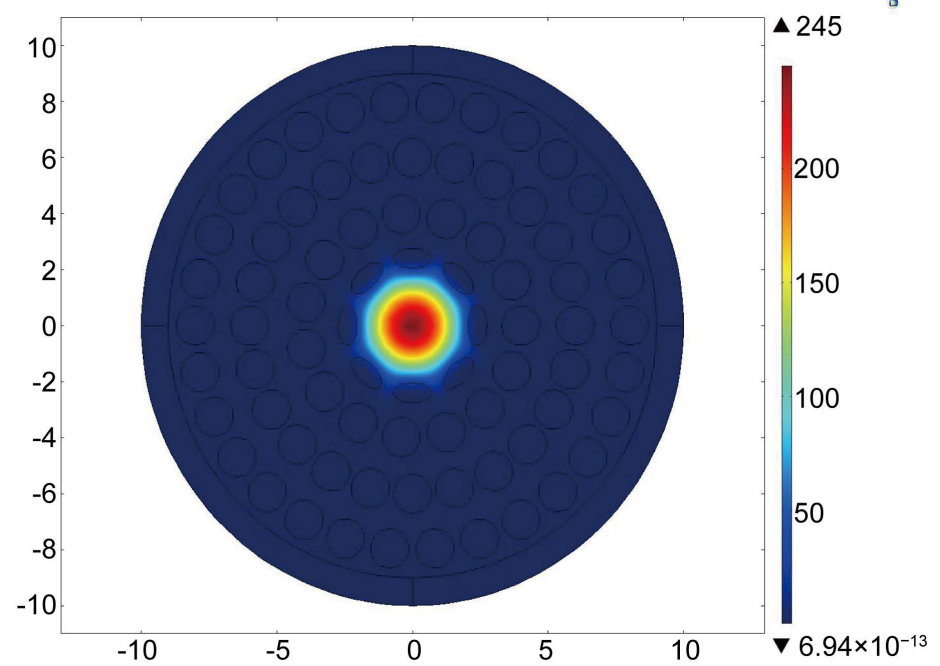

(b)

Figure 2. Analyzed circular PCF model in COMSOL Multiphysics: (a) Transverse geometry; and (b) Fundamental mode field of the Circular PCF geometry.

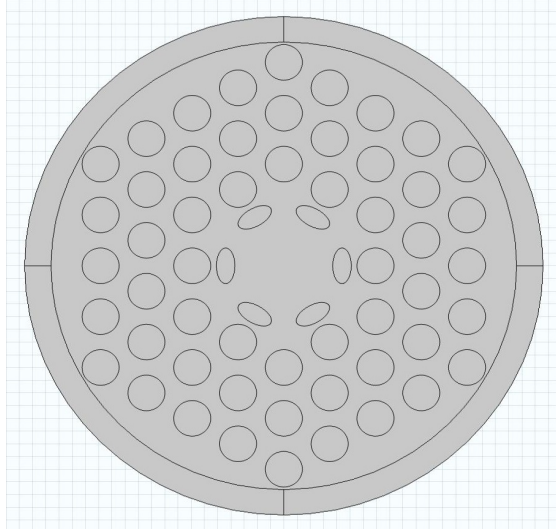

(a)

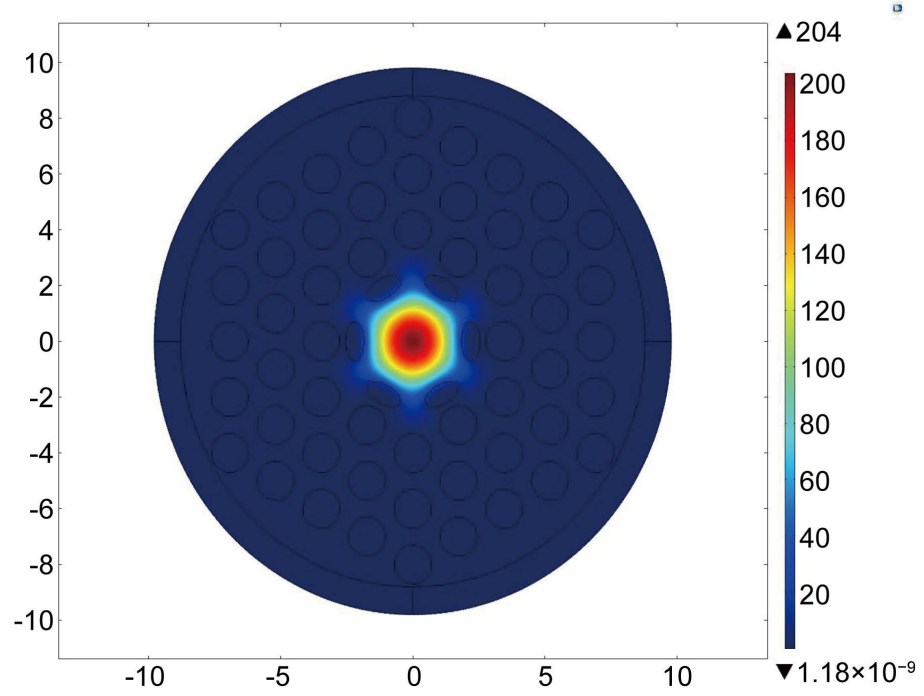

(b)

Figure 3. Analyzed hexagonal PCF model in COMSOL Multiphysics: (a) Transverse geometry; and (b) Fundamental mode field of the Hexagonal PCF geometry.

Figure 5 illustrated the propagation constant variation with respect to wavelength from $800 \mathrm{~nm}$ to $1600 \mathrm{~nm}$ for square, circular and hexagonal geometry. The propagation constant decreases with the increases of wavelength for all the three structures and the variation of propagation constant among the three structures are so close that we can't differentiate the three curves from the figure. For all the three structures the highest propagation constant is at wavelength $800 \mathrm{~nm}$ and lowest value is at $1600 \mathrm{~nm}$.

Figure 6 illustrated the confinement loss variation with respect to wavelength from $800 \mathrm{~nm}$ to $1600 \mathrm{~nm}$ for square, circular and hexagonal geometry. The Confinement 


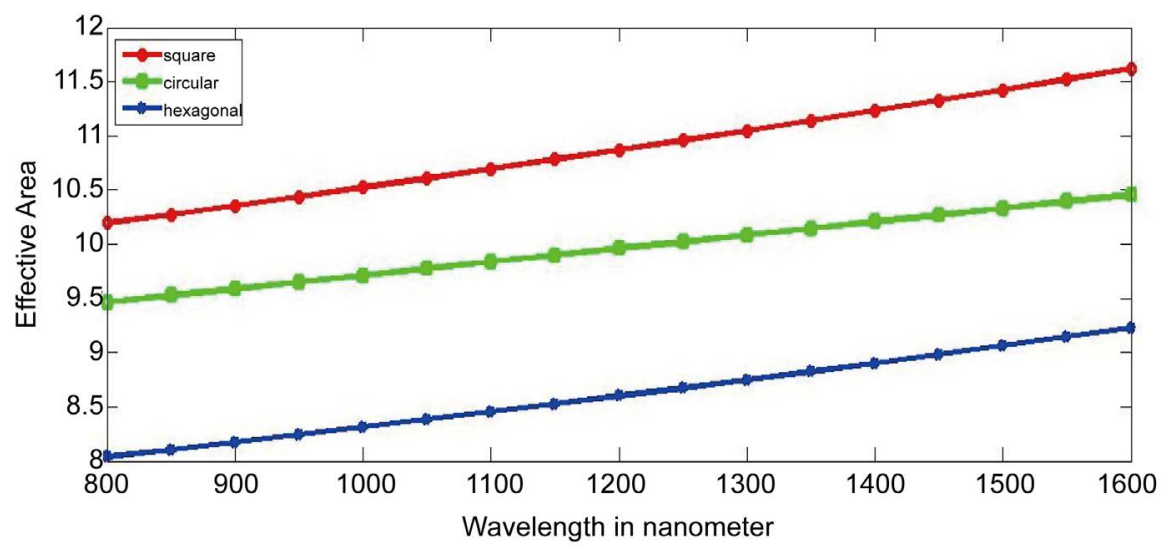

Figure 4. Effective area variation with respect to wavelength from $800 \mathrm{~nm}$ to $1600 \mathrm{~nm}$ for square, circular and hexagonal geometry.

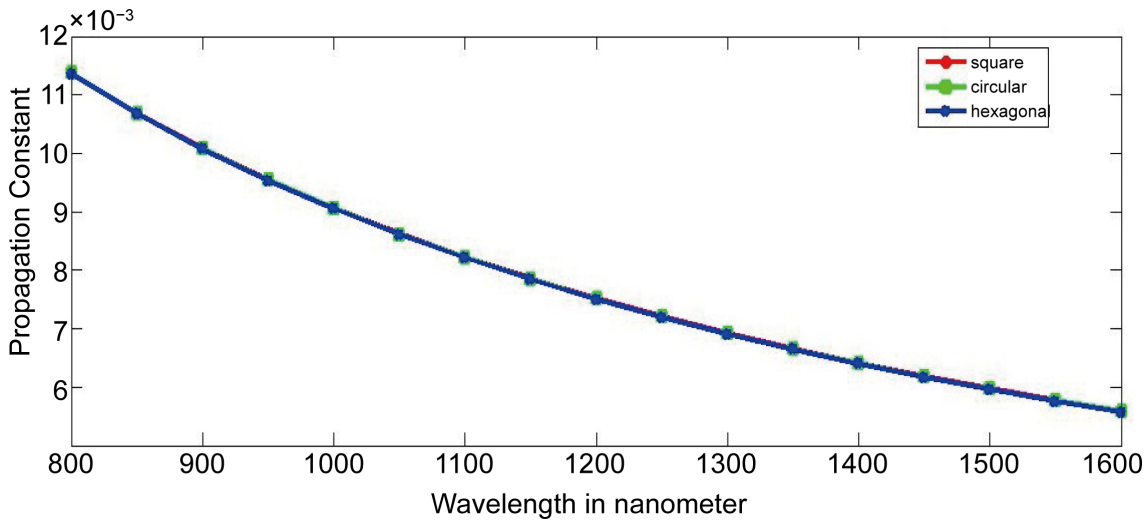

Figure 5. Propagation constant variation with respect to wavelength from $800 \mathrm{~nm}$ to 1600 $\mathrm{nm}$ for square, circular and hexagonal geometry.

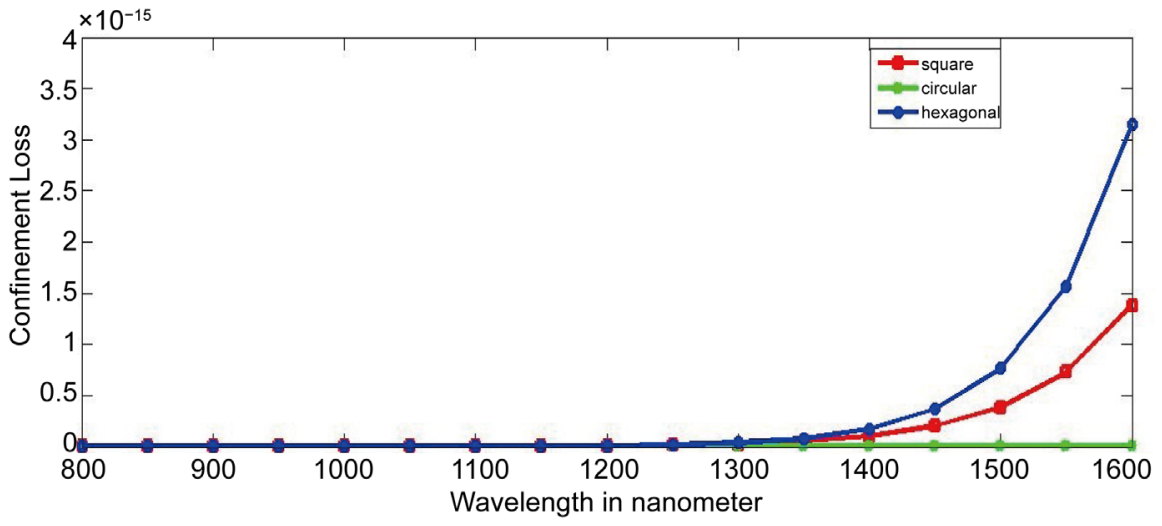

Figure 6. Confinement loss variation with respect to wavelength from $800 \mathrm{~nm}$ to 1600 $\mathrm{nm}$ for square, circular and hexagonal geometry.

loss is approximately zero at wavelength ranges from $800 \mathrm{~nm}$ to $1250 \mathrm{~nm}$ and after that, it increases with the increases of wavelength for all the three structures. Large confinement loss is seen for hexagonal PCF geometry and low confinement loss is seen for circular PCF geometry. For the square PCF geometry 


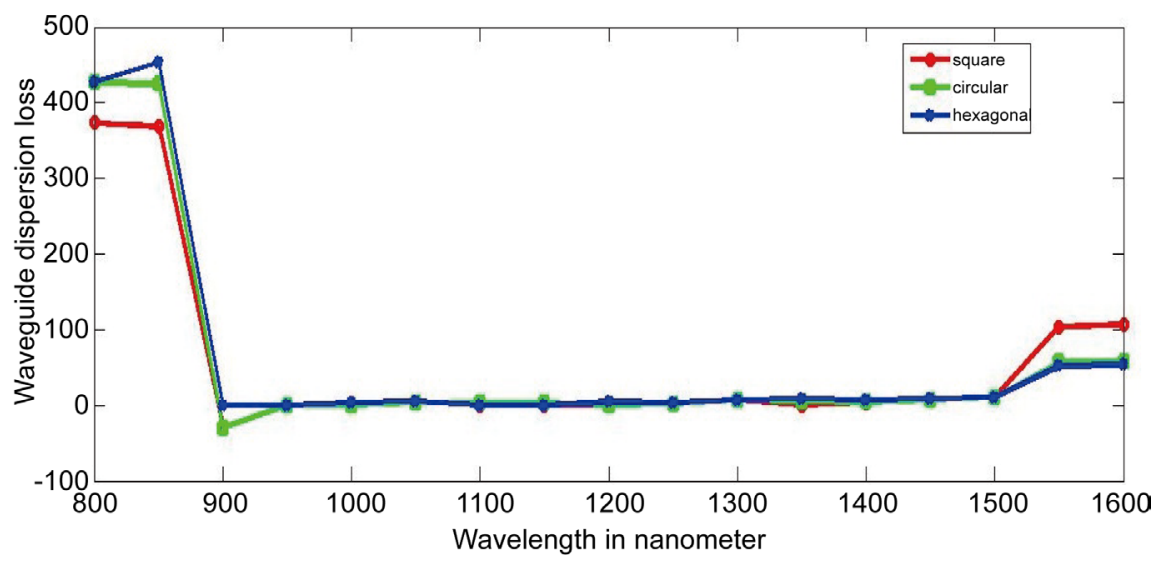

Figure 7. Waveguide dispersion variation with respect to wavelength from $800 \mathrm{~nm}$ to $1600 \mathrm{~nm}$ for square, circular and hexagonal geometry.

the Confinement loss is nearly $1.592 \times 10^{-16} \mathrm{~dB} / \mathrm{km}$, for the circular PCF geometry the confinement loss is $6.88 \times 10^{-16} \mathrm{~dB} / \mathrm{km}$, for the hexagonal PCF geometry, the confinement loss is $0.58 \times 10^{-16} \mathrm{~dB} / \mathrm{km}$ in wavelength $1550 \mathrm{~nm}$.

Another very significant optical parameter is waveguide dispersion. Figure 7 illustrated the waveguide dispersion variation with respect to wavelength from $800 \mathrm{~nm}$ to $1600 \mathrm{~nm}$ for square, circular and hexagonal geometry. Waveguide dispersion is high initially for all the three PCFs and after that, it changes abruptly. Approximately zero waveguide dispersion is achieved from $900 \mathrm{~nm}$ to 1500 $\mathrm{nm}$ for all the three PCF structures. The waveguide dispersion is approximately $103.333 \mathrm{ps} /(\mathrm{nm} \cdot \mathrm{km}), 56.646 \mathrm{ps} /(\mathrm{nm} \cdot \mathrm{km}), 51.66 \mathrm{ps} /(\mathrm{nm} \cdot \mathrm{km})$ at wavelength 1550 nm.

We have got only negative dispersion for circular PCF structure and it is -30.354 $\mathrm{ps} /(\mathrm{nm} \cdot \mathrm{km})$ at the wavelength of $900 \mathrm{~nm}$.

\section{Conclusion}

In this research work, four-layer square, circular and hexagonal photonic crystal fiber have been designed. The research is focused on the variation of optical properties like propagation constant, confinement loss, effective area, and waveguide dispersion by varying the structure of PCFs. The simulation results have shown that the effective area is small for hexagonal PCF geometry and large for square PCF geometry. From the analysis, the Confinement loss is approximately zero at wavelength ranging from $800 \mathrm{~nm}$ to $1250 \mathrm{~nm}$ and approximately zero waveguide dispersion is achieved from $900 \mathrm{~nm}$ to $1500 \mathrm{~nm}$ for all the three PCF structures. Besides, negative dispersion, approximately $-30.354 \mathrm{ps} /(\mathrm{nm} \cdot \mathrm{km})$, is also achieved for circular PCF structure at the wavelength of $900 \mathrm{~nm}$.

\section{Future Work}

In this paper, simulation is carried out for square, circular, and hexagonal photonics crystal fiber geometry to analysis the effective area, confinement loss, waveguide dispersion, propagation constant etc. In the next work, relative sensitivity, 
coupling length and birefringence for different PCF geometries will be taken into account during simulation. Finally, the PCF fabrication process will be studied and the proposed PCF structures will be fabricated.

\section{References}

[1] Razzak, S.A., and Namihira, Y. (2008) Tailoring Dispersion and Confinement Losses of Photonic Crystal Fibers Using Hybrid Cladding. Journal of Lightwave Technology, 26, 1909-1914. https://doi.org/10.1109/JLT.2008.922323

[2] Wong, G.K.L., Chen, A.Y.H., Ha, S.W., Kruhlak, R.J., Murdoch, S.G., Leonhardt, R., Harvey, J.D. and Joly, N.Y. (2005) Characterization of Chromatic Dispersion in Photonic Crystal Fibers Using Scalar Modulation Instability. Optics Express, 13, 8662-8670. https://doi.org/10.1364/OPEX.13.008662

[3] Quimby, R.S. (2006) Photonics and Lasers: An Introduction. John Wiley \& Sons, Hoboken. https://doi.org/10.1002/0471791598

[4] Chen, D., Vincent Tse, M.L. and Tam, H.Y. (2010) Optical Properties of Photonic Crystal Fibers with a Fiber Core of Arrays of Subwavelength Circular Air Holes: Birefringence and Dispersion. Progress in Electromagnetics Research, 105, 193-212. https://doi.org/10.2528/PIER10042706

[5] Razzak, S.A., Namihira, Y., Begum, F., Kaijage, S., Kinjo, T., Nakahodo, J., Miyagi, K. and Zou, N. (2007) Decagonal Photonic Crystal Fibers with Ultra-Flattened Chromatic Dispersion and Low Confinement Loss. Optical Fiber Communication and the National Fiber Optic Engineers Conference 2007, OFC/NFOEC 2007, Anaheim, 25-29 March 2007, 1-6. https://doi.org/10.1109/OFC.2007.4348389

[6] Guo, S., Wu, F., Albin, S., Tai, H. and Rogowski, R.S. (2004) Loss and Dispersion Analysis of Microstructured Fibers by Finite-Difference Method. Optics Express, 12, 3341-3352. https://doi.org/10.1364/OPEX.12.003341

[7] Saitoh, K., Koshiba, M., Hasegawa, T. and Sasaoka, E. (2003) Chromatic Dispersion Control in Photonic Crystal Fibers: Application to Ultra-Flattened Dispersion. $O p$ tics Express, 11, 843-852. https://doi.org/10.1364/OE.11.000843

[8] Hansen, K.P. (2003). Dispersion Flattened Hybrid-Core Nonlinear Photonic Crystal Fiber. Optics Express, 11, 1503-1509. https://doi.org/10.1364/OE.11.001503

[9] Steel, M.J. and Osgood, R.M. (2001) Elliptical-Hole Photonic Crystal Fibers. Optics Letters, 26, 229-231. https://doi.org/10.1364/OL.26.000229

[10] Habib, M.S., Hasan, M.I., Razzak, S.M.A., Mahmud, R.R. and Namihira, Y. (2013) Microstructure Holey Fibers as Wideband Dispersion Compensating Media for High Speed Transmission System. Optik-International Journal for Light and Electron Optics, 124, 4984-4988. https://doi.org/10.1016/j.ijleo.2013.03.128

[11] Priya, K.R., Raja, A.S. and Sundar, D.S. (2016) Design of a Dual-Core Liquid-Filled Photonic Crystal Fiber Coupler and Analysis of Its Optical Characteristics. Journal of Optical Technology, 83, 569-573. https://doi.org/10.1364/JOT.83.000569

[12] Russell, P.S.J. (2006) Photonic-Crystal Fibers. Journal of Lightwave Technology, 24, 4729-4749. https://doi.org/10.1109/JLT.2006.885258

[13] Kim, S.E., Kim, B.H., Lee, C.G., Lee, S., Oh, K. and Kee, C.S. (2012) Elliptical Defected Core Photonic Crystal Fiber with High Birefringence and Negative Flattened Dispersion. Optics Express, 20, 1385-1391. https://doi.org/10.1364/OE.20.001385

[14] Saitoh, K., Florous, N. and Koshiba, M. (2005) Ultra-Flattened Chromatic Dispersion Controllability using a Defected-Core Photonic Crystal Fiber with Low Confinement Losses. Optics Express, 13, 8365-8371. 
https://doi.org/10.1364/OPEX.13.008365

[15] Matsui, T., Zhou, J., Nakajima, K. and Sankawa, I. (2005) Dispersion-Flattened Photonic Crystal Fiber with Large Effective Area and Low Confinement Loss. Journal of Lightwave Technology, 23, 4178-4183.

https://doi.org/10.1109/JLT.2005.858250

[16] Chung, K.W., Kim, S. and Yin, S. (2003) Design of a Highly Nonlinear Dispersion-Shifted Fiber with a Small Effective Area by Use of the Beam Propagation Method with the Gaussian Approximation Method. Optics Letters, 28, 2031-2033. https://doi.org/10.1364/OL.28.002031

[17] Ferrando, A., Silvestre, E., Miret, J.J. and Andres, P. (2000) Nearly Zero Ultraflattened Dispersion in Photonic Crystal Fibers. Optics Letters, 25, 790-792.

https://doi.org/10.1364/OL.25.000790

[18] Roberts, P.J., Couny, F., Sabert, H., Mangan, B.J., Williams, D.P., Farr, L., Mason, M.W., Tomlinson, A., Birks, T.A., Knight, J.C. and Russell, P.S.J. (2005) Ultimate Low Loss of Hollow-Core Photonic Crystal Fibres. Optics Express, 13, 236-244. https://doi.org/10.1364/OPEX.13.000236

[19] Florous, N., Saitoh, K. and Koshiba, M. (2006) The Role of Artificial Defects for Engineering Large Effective Mode Area, Flat Chromatic Dispersion, and Low Leakage Losses in Photonic Crystal Fibers: Towards High Speed Reconfigurable Transmission Platforms. Optics Express, 14, 901-913.

https://doi.org/10.1364/OPEX.14.000901 